\section{The Second Decade: What Can We Do About THE ADOLESCENT BRAIN?}

\author{
By Catherine Sebastian
}

With a recent UNICEF report showing our youth to be the unhappiest in the industrialised world (UNICEF, 2007; BBC, 2007), the time has come to seriously review the way in which our society treats its young people. It is debatable whether our overarching motivation to do so is an altruistic one (to improve lives), or one of selfpreservation (fear of $A$ Clockwork Orange-style random violence). Both are, at this point, equally compelling.

In the past decade, cognitive neuroscientists have joined the attempts of psychologists, social workers, youth workers and educators to understand behaviour during the teenage years. Cognitive neuroscience is a relatively young discipline which attempts to link cognitive processes such as memory, attention and decision-making, with activity in the brain (see Gazzaniga, 1999 for an overview). Brain activity is measured indirectly by looking at behaviour, or increasingly using brain imaging and recording methods. Even more recently, social cognitive neuroscience has begun to look at the neural underpinnings of processes such as emotion and interpersonal interaction. As a result, it has been fascinating to discover a basis in the brain for phenomena such as the role of emotion in decision-making; phenomena which until now have been the preserve of philosophy and literature.

This article will look at evidence of neural differences between adolescents (between the ages of around 10 and 16) and adults, and how this relates to differences in behaviour. There may be a link between changes in the brain during adolescence and stereotypically 'adolescent' behaviour such as increased risk-taking, poor self-control, and emotional instability. But even if you could quantify all the causes of these behaviours at a neural level, what implications would this have? Could it lead to effective interventions at the interpersonal or societal level? Perhaps the cognitive neuroscience of adolescence is merely an academic exercise. But before we can look at implications, I will review the key findings from the neuroscience literature.

\section{Structure of the Teenage Brain}

The human brain is composed of around 100 billion nerve cells or 'neurons'. The cell bodies comprise the 'grey matter', and are heavily interconnected via fibres known as 'axons'. These fibres are coated in a fatty substance called myelin which acts as an insulator, enabling electrical signals to be transmitted more efficiently. The insulated axons bundle together to form 'white matter', much like a bundle of electrical cables. While it was long assumed that the brain was fairly unchanging after early childhood, it is now known that both white and grey matter continue to develop well into our twenties. Indeed, some areas of the brain continue to manufacture new neurons to replace old ones throughout the lifespan.

Particularly intriguing are the changes that have been found to occur at the onset of puberty. Although the relationship between pubertal hormone release and brain development is not fully established, it is known that a cascade of neural changes is initiated early in adolescence. This drives the transformation of the brain from that of a child to that of an adult. This makes evolutionary sense - as the body reaches sexual maturity, the drive to leave home and find a mate must be matched by a brain that is well-equipped to negotiate the risks and challenges this poses.

Studies (e.g. Sowell, Thompson, Holmes, Jernigan, and Toga, 1999; Paus et al., 1999) have shown that the volume of white matter increases steadily throughout adolescence, particularly in the frontal and parietal cortices; regions of the brain associated with complex abilities such as planning, paying attention, and interacting with other people. This means that the capacity of the brain to conduct signals efficiently from one region to another increases throughout adolescence. At the same time, changes in grey matter are also occurring, although these are more complex and vary between brain regions. As a general rule, grey matter density varies in an 'inverted U shape', i.e. volume initially increases and then gradually decreases again. This is thought to reflect changes in the number of connections or 'synapses' between neurons. At the onset of puberty (around the age of 11 in girls and 12 in boys), there is a proliferation of synapses. However, during the following important years, redundant synapses are pruned away (see Paus, 2005 for a review). This results in more efficient, faster adult networks, capable of more sophisticated information processing. It is an important principle of the brain that more 
isn't always better: it's the quality, not quantity, of the connections between brain cells that counts.

\section{Functioning of the Teenage Brain}

So what do these structural alterations to the adolescent brain mean for behaviour? First, it's important to note that areas undergoing the most profound restructuring at this time include some of our most sophisticated hardware. Most of the brain is fully developed by early childhood, and the behavioural differences between adults and adolescents are actually subtle (although it might not always seem that way!). For example, teenagers have no trouble making the complex but automatic visual calculations necessary to work out when an approaching car will reach their location. However, a teenager may decide that they have time to cross the road before it gets there, whereas an adult might decide to play it safe and wait. This is of course a generalisation, but it illustrates that the most profound differences between adults and adolescents occur at the decision-making, or executive, levels of processing. Anatomically, the brain areas subserving these functions are located in the prefrontal cortex (PFC), situated at the very front of the brain. The PFC is larger in humans, relative to body size, than in most other animals, and is thought to control many of our unique abilities. These include executive abilities such as forward planning, self-control, reasoning, and decision-making, as well as social cognitive abilities such as mentalising (the ability to infer what someone thinks, feels, or believes).

Experiments have revealed some quite surprising differences between adults and adolescents on tasks involving these areas. For example, the Iowa Gambling Task is often used to assess the functioning of the ventromedial PFC, a region at the very front of the PFC just above the eye sockets. It is thought to be involved in the evaluation of rewards for the purposes of making decisions, and is often studied using gambling tasks that provide an explicit measure of the decisions people make in the face of specific risks and rewards. In the Iowa Gambling Task, four decks of cards are laid out in front of the participant: cards represent either a monetary reward or loss, and participants must choose from the decks with the aim of maximising the amount of money they gain over time. Two of the decks contain cards offering high rewards but also heavy losses, while the other two offer more modest rewards but also smaller losses. The decks are rigged so that the latter two decks will yield the greatest gain over many trials. Normal adults start by sampling all four decks, but gradually shift their choices to the 'good' decks. People with damage to the ventromedial PFC do not learn this, and continue to take risks on the 'bad' decks.

Crone and van der Molen (2004) gave this task to people aged from 6 to 25 years. They found that although all but the youngest subjects showed some shift towards the good decks over time, the 1015 year olds took a lot longer than the young adults to make the shift. Even at the end of the experiment they were only choosing the good decks $55-60 \%$ of the time - well below adult levels, and similar to patients with damage to the ventromedial PFC. It could be that the immaturity of this brain region underpins findings from studies of risk-taking behaviour in the real world, which show that adolescents are more likely than adults to engage in risky activities such as unprotected sex, reckless driving, and experimentation with drugs. If, as the results suggest, young people are less good at anticipating the outcome of events, perhaps they are unable to accurately appraise the risk levels when faced with a real-life choice.

But this is not the whole story. Other studies have suggested that teenagers can accurately appraise risk to the same degree as adults in a controlled experimental situation, but when faced with a realworld situation this does not necessarily translate into a sensible decision. Interaction with the environment is crucial here, and aberrant decision-making in adolescence is often mediated by the presence of peers. Gardner and Steinberg (2005) put teenagers and adults in a driving simulation game. Half the subjects played alone, and the other half played with two friends watching and giving advice. Both teenagers and adults took few risks when driving alone (e.g. driving through a yellow light instead of stopping). However, in the presence of peers, the teenagers, but not the adults, took many more risks. This seems to reflect what we intuitively know from our own experience. But why should this be the case? Is it a purely social effect, reflecting the growing importance of respect from peers? Is it due to hormones? Or could there be an interaction between the development of brain areas involved in decisionmaking and those involved in social cognition, e.g. knowing what others are thinking about you, and indeed awareness of your own thoughts and intentions?

Social cognition in adolescence has been neglected until recently, but our lab and others are now investigating processes such as taking another's point of view, processing of complex emotions, 
emotional/neural reactivity to stressful social situations such as ostracism, and knowledge about our own and others' intentions and thoughts. For example, a recent study by Blakemore et al. (in press) used functional magnetic resonance imaging (fMRI) to look at the brain areas activated when adolescents and adults make decisions about either intentional causality (what you would choose to do in a given situation) or physical causality (the consequences of a natural event such as heavy rain, that does not involve a human component). They found that when asked to think about intentions, a larger area of the medial PFC was activated in adolescents compared with adults. This is another brain region known to undergo substantial pruning during adolescence, and is an area implicated in thinking about the self and other people. The high level of activation may reflect processing by networks that are still relatively inefficient at this kind of social cognition. In addition, adults activated a different brain area, the right superior temporal sulcus, more than adolescents during the intentional causality task. This area is commonly activated in mentalising tasks and in predicting other people's behaviour. It may be that the shift towards using this region reflects the increasing maturity of the mentalising network, although it should be noted that adults and adolescents were using very similar networks, just with subtle differences. Implications for behaviour are still fairly speculative, but it is certainly interesting that adults and adolescents are processing social and self-related information using different neural strategies.

Neural control of automatic or impulsive behaviour is another issue to consider when attempting to explain adolescent behaviour. Several studies have looked at the ability of adolescents to inhibit a habitual response when the task requires it. For example, Tamm, Menon, and Reiss (2002) used a 'Go/No-Go' task, in which participants had to press a button whenever they saw a letter ('Go' stimulus), unless the letter was an $\mathrm{X}$ ('No-Go' stimulus). Although this is a simple task, it illustrates a very important executive function - the ability to control our behaviour in a flexible and taskdependent manner. Adolescents did not differ from adults in terms of performance on the task, but the brain activation as measured with fMRI revealed that adolescents activated more of the PFC, and activated it to a greater degree, to achieve the same effect. This suggests that it is more effortful for adolescents to suppress their impulses and respond to even simple demands on cognitive control. If we combine this finding with the evidence above about risk taking and social processing (and consider that there are almost certainly interactions between these functions that we have not yet studied), it can be seen that adolescents are often facing adult decisions with a brain that is not fully equipped to deal with them.

\section{Implications}

In some sense British society already deals effectively with the differences between adults and adolescents. For example, important civil liberties such as suffrage are not granted until the age of 18, and potentially risky activities such as driving, smoking, and drinking alcohol are also subject to an age restriction. Implicit in these restrictions is the idea that a certain level of maturity and responsibility is required for appropriate decisions to be taken on these issues, and that by virtue of reaching post-adolescence, the required level is generally attained.

So British society is clear on what it doesn't want adolescents to be doing, but does it have a realistic outlook on how it does want them to be spending their time? I would argue that many adolescents spend several of their teenage years in limbo, dumped at school with little to fulfil the four basic emotional needs identified by social psychologists: control over one's life, a meaningful existence, high self-esteem, and belonging. Friends can certainly provide a sense of belonging and high self-esteem, but many young people face bullying, racism, and discrimination, not just from peers, but also from adults who have become so alienated by teenage antisocial behaviour that they apply a policy of universal hostility.

I would also argue that the way our society is structured largely denies the possibility for teenagers to have a meaningful existence, nor are they able to feel in control of their lives. Poverty, low expectations, and poor educational experience deprive many of aspirations for the future, especially in our individualistic society in which success is measured by career advancement and material wealth. Teenagers are often also unable to carve a niche for themselves, academically or otherwise, due to a restrictive curriculum, a 'one size fits all' educational policy, and underinvestment in affordable activities outside school. It is interesting here to consider education and youth services in other industrialised nations that scored far higher on the happiness of young people than the UK in the UNICEF report (UNICEF, 2007). In other industrialised nations such as the Netherlands and Sweden there is less emphasis on exams, a wider range of options other than the classic 'academic route', and more emphasis on the 
development of social skills before children reach adolescence. Findings from neuroscience research on the adolescent brain are not going to change society, but they might be able to provide clues about how social and educational policies interact with the developing brain during adolescence, and thus support links between policy and measures of adolescent experience.

More immediately, we might ask whether neuroscientific findings can be used practically to improve the experience of the average teenager. The brain is a complex structure that develops in tandem with the rest of the body, and in response to the external environment. Therefore, except in the case of psychiatric disorder, it would not be wise to intervene with medication, even if it were to make the experience of adolescence less turbulent. In some sense, adolescence is meant to be a tough time. But parents, educators, and teenagers themselves should be made aware of the role that brain development will play in behaviour. Often, just knowing the reasons for something can help us to feel more in control, and it may promote understanding between different age groups. If young people know why they are more susceptible to peer pressure, or why they feel like taking risks sometimes, or why they feel so different from adults, this might at least go some way towards helping them feel less alone and make them better equipped to deal with decisions they have to face.

In addition to educating people in this way, it may be possible to develop behavioural strategies to train adolescents in 'weak' areas, by giving them progressively harder computerised exercises in domains such as cognitive control, or thinking about other minds, or decision tasks requiring an individual to weigh and compare different options in order to make a choice. Or, particularly for social cognition, workshops and discussion groups could be provided in which young people discuss a situation from both their own and another's perspective in order to refine mentalising abilities. These abilities could then be extended by introducing schemes that give young people a sense of social responsibility, e.g. mentoring for younger children, or charity work in the community. Whether such tasks would generalise to teenagers' lives in the real-world is debatable, as is the assumption that this sort of practice would translate to more 'adult' activity at the neural level. Perhaps time just has to take its course. There is also the social argument that teenagers are meant to experiment and make mistakes, and that it puts even more pressure on them to ask them to behave like responsible adults. Yet people are taking important decisions at a young age (e.g. when to have sex, whether to use contraception, whether to drink or take drugs, see UNICEF, 2007), and something needs to be done to ensure that they are equipped to make them. Although neuroscience cannot address these issues alone, it may well be of use in combination with the efforts of other fields such as psychology, education, and social work.

\section{Conclusions}

There is clear evidence that the adolescent brain is very different to that of an adult, and that substantial restructuring of the prefrontal cortex needs to occur during these important years. Cognitive and social cognitive neuroscience are now starting to reveal how differences in brain structure and function relate to typically 'adolescent' behaviours such as risk taking, susceptibility to peer pressure, poor perspectivetaking, emotional instability, and more effortful selfcontrol. But there is still a long way to go. What are the interactions between these behaviours and the environment? For example, do teenagers find it more difficult to regulate and control the feelings they experience during negative social experiences (e.g. bullying and ostracism), and might this then make them more likely to conform to peer pressure? Until we know more about how the discoveries made in the lab relate to the everyday experiences of adolescents, it will be difficult to put our research to practical use. Nevertheless, this emerging field provides a new angle on an increasingly thorny problem: parents, educators and policy makers should not just consign these findings to academic oblivion, but should work with scientists to find new applied solutions.

(c) Catherine Sebastian, 2007

PhD 1, Psychology (Perspectives on the Self from Adolescence and Asperger Syndrome)

\section{References}

BBC. UK is accused of failing cbildren. Retrieved April 3, 2007, from http://news.bbc.co.uk/1/hi/uk/6359363.st $\mathrm{m}$.

Blakemore, S-J., den Ouden, H. E. M., Choudhury, S. \& Frith, C. Adolescent development of the neural circuitry for thinking about intentions. In press in Social Cognitive and Affective Neuroscience. 
Crone, E. A., \& van der Molen, M. W. (2005). Developmental Changes in Real Life Decision Making: Performance on a Gambling Task Previously Shown to Depend on the Ventromedial Prefrontal Cortex. Developmental Neuropsychology, 25(3), 251-279.

Gardner, M., \& Steinberg, L. (2005). Peer influence on risk taking, risk preference, and risky decision making in adolescence and adulthood: An experimental study. Developmental Psychology, 41(4), 625-635.

Gazzaniga, M. S. (Ed.). (1999). The New Cognitive Neurosciences. Cambridge: MIT.

Paus, T. (2005). Mapping brain maturation and cognitive development during adolescence. Trends in Cognitive Sciences, 9(2): 60-68.

Paus, T., Zijdenbos, A., Worsley, K., Collins, D. L., Blumenthal, J., Giedd, J. N., Rapoport, J. L., \& Evans, A. C. (1999). Structural maturation of neural pathways in children and adolescents: in vivo study. Science, 283, 1908-1911.

Sowell, E. R., Thompson, P. M., Holmes, C. J., Batth, R., Jernigan, T. L., Toga, A. W. (1999). Localizing age-related changes in brain structure between childhood and adolescence using statistical parametric mapping. Neuroimage 9(6): 587-97.

Steinberg, L. (2005). Cognitive and affective development in adolescence. Trends in Cognitive Sciences, 9(2): 69-74.

Tamm, L., Menon, V., \& Reiss, A. L. (2002). Maturation of brain function associated with response inhibition. Journal of the American Academy of Child and Adolescent Psychiatry, 41, 1231-1238.

Trends in Cognitive Sciences, 9(2), 60-68.

UNICEF. An overview of child well-being in rich countries. Retrieved April 3, 2007, from http://www.unicef-

icdc.org/publications/pdf/rc7_eng.pdf. 